International Journal of Biomedicine I June 2019 - Volume 9, Issue Suppl_1:

Abstracts From the Second Russian International Conference "Cryo-electron microscopy 2019: achievements and prospects"

ORAL ABSTRACT PRESENTATIONS

SESSION TITLE: COMPLEX AND EMERGING TECHNIQUES IN STRUCTURAL BIOLOGY

DOI: 10.21103/IJBM.9.Suppl_1.OR24

\title{
Abstract OR-24: Detection and Characterization of Extracellular Vesicles in Transmission Electron Microscopy by Convolutional Neural Network
}

Igor Nikishin, Dmitry Bagrov, Elena Tchevkina, Gleb Skryabin, Ruslan Dulimov

Department of Biology, Lomonosov Moscow State University, Moscow, Russia

Background: Extracellular vesicles (EVs) are produced by almost all known organisms and released or secreted from cells. EVs particles are lipid-based particles with size ranging from 30 to 1000 $\mathrm{nm}$. Transmission Electron Microscopy (TEM) is the most convenient method for imaging of EVs. The size measurement of the EVs on the TEM images can be automated and accelerated using convolutional neural networks (CNN). Our approach is to use a CNN that transforms a TEM image to a probabilistic map that indicates where extracellular vesicles particles are located. Our method is based on supervised learning that requires both the input images and their corresponding annotations.

Methods: We used EVs isolated from ascitic fluids from ovarian cancer patients by ultracentrifugation. The TEM images were obtained using negative staining. The software development included four steps: data augmentation and marking datasets using VGG Image Annotator (VIA) software (Oxford, England); neural network education; particle detection and features extractions. Mask R-CNN was chosen as the core $\mathrm{CNN}$ for object detection and instance segmentation using Keras and TensorFlow.

Results: Mask RCNN Neural Network was educated using more than 2000 particles. The EVs were counted, and their size was measured (area, effective diameter, square). We added the option of manual checking the results. After processing, the software calculates the key parameters of each EV and provides the results as a table for further statistical analysis.

Conclusion: Our approach helps to analyze each TEM image of vesicles approximately 10 times faster than by handpicking of single EVs. Since the CNN method is highly flexible, similar convolutional networks could be adapted to detect other particles by learning from annotated datasets.

Key Words: extracellular vesicles • TEM • neural networks

Sources of Funding: This work was supported by the Russian Fund for Basic Research, project № 17-0000166. The electron micrographs were made at User Facilities Center of Lomonosov Moscow State University under financial support of Ministry of Education and Science of Russian Federation. 
International Journal of Biomedicine. 2019;9 Suppl 1: S16. doi: 10.21103/IJBM.9.Suppl_1.OR24 C2019 International Medical Research and Development Corporation 